\title{
EDITORIAL
}

\section{A special issue on photonic signal processing}

\author{
Xinliang ZHANG (凶) ${ }^{1}$, Jianping CHEN ${ }^{2}$ \\ 1 Wuhan National Laboratory for Optoelectronics, Huazhong University of Science and Technology, Wuhan 430074, China \\ 2 Department of Electronic Engineering, Shanghai Jiao Tong University, Shanghai 200240, China
}

(C) Higher Education Press and Springer-Verlag Berlin Heidelberg 2011

Photonic signal processing has been receiving increasing attention for about fifteen years. It would be enabling technology for next generation large capacity optical networks because it can avoid electronics bottleneck and decrease power consumption greatly. There are two main areas related to photonic signal processing. One topic is all-optical signal processing for digital bit sequence in optical network, such as all-optical wavelength conversion, alloptical logic operation, all-optical 3R regeneration, all-optical clock recovery, all-optical buffer, etc. The other topic is microwave photonic signal processing, such as microwave photonic filters, arbitrary waveform generation, RF signal generation, UWB signal generation, etc. However, there are still many problems to be solved before these functions could be used in optical networks. Researchers all over the world are concentrating on realizing photonic signal processing functions with some characteristics such as high operation speed and large bandwidth, flexible and multifunctional, potential integration.

This special issue on photonic signal processing of Frontiers of Optoelectronics in China has collected review and research articles by a number of Chinese and international experts, especially from the research group sponsored by the National Basic Research Program of China (Grant No. 2011CB301700). There are seventeen excellent papers in this special issue including 4 review articles and 13 research articles.

Prof. Yong Liu is a well known specialist in the field of photonic signal processing, and he reviewed some signal processing functions with nonlinear effects in semiconductor optical amplifiers. A wonderful review paper on slow light with silicon photonic crystals was given by Prof. Eric Cassan from University Paris-Sud, and he discussed the challenges, hot research topics and future trends in this field. Prof. Xinwan Li from Shanghai Jiao Tong University reviewed some micro/nanostructure photonic devices for photonic signal processing, such as micro/nanofiber based devices and silicon waveguide based devices. Dr. Enming Xu reviewed some results on microwave photonic filters with semiconductor optical amplifiers.

Moreover, there are 13 research articles focusing on photonic signal processing. An invited paper on compact silicon and hybrid plasmonic waveguides used for signal processing was presented by Prof. Yikai Su from Shanghai Jiao Tong University. Prof. Xianmin Zhang's group presented a novel method for compensating dispersion effect in phase modulated radio over fiber links using an optical carrier Brillouin processing technique. Recent progress on slow light with cascaded microring resonators was reported by Prof. Jinzhong Yu's group. Yu Ji from Prof. Jian Wu's group demonstrated $40 \mathrm{GHz}$ phase stable optical pulses with pulse-width of $1.88 \mathrm{ps}$, extinction ratio larger than 20 $\mathrm{dB}$, the timing jitter of $57 \mathrm{fs}$ and signal-to-noise ratio of $32.8 \mathrm{~dB}$. Prof. Caiyun Lou and her colleagues also reported some research progress on optical signal processing functions with semiconductor optical amplifiers and delay interferometers. Some results on all-optical clock recovery with magnetically controllable fiber optical parametric oscillator were demonstrated by Prof. Baojian Wu. Ping Zhao from Xinliang Zhang's group presented a novel method for fabricating silica micro/nanofiber.

Also, there are some theoretical simulation results in this special issue. Xinying Li from Prof. Nan Chi's group analysed the impact of quadrature imbalance in the presence of frequency offset in an optical coherent offset quadrature phase-shift keying receiver. Prof. Guangqiong Xia and her colleagues analysed the impacts

Received July 2, 2011

E-mail: xlzhang@mail.hust.edu.cn 
of mismatched intrinsic parameter on leader-laggard chaos synchronization between two mutually coupled vertical-cavity surface-emitting lasers. Xiaowei Dong from Beijing Jiaotong University reported some theoretical analysis results on complex long-period-grating-assisted-coupler. Xi Huang from Xinliang Zhang's group gave a theoretical analysis on band structure calculation methods for quantum well semiconductor optical amplifiers. Zheng Zhang from Xinliang Zhang's group proposed and simulated a simple scheme of all-optical format conversion from non-return-to-zero differential phase-shift keying to return-to-zero differential phase-shift keying. Yujie Zhou from Prof. Junqiang Sun's group gave a theoretical analysis on the mode overlap of propagated waves in the direct bonded periodically poled $\mathrm{MgO}$ doped lithium niobate (PPMgLN) ridge waveguide.

We would like to express our great thanks to these contributors of this special issue. These articles showcase the exciting research results in the field of photonic signal processing. We have more reasons to believe that photonic signal processing functions would be applied in practical and commercial optical networks in the near future.

Xinliang Zhang and Jianping Chen

The Guest Editors of Special Issue on Photonic Signal Processing 